\title{
An Intelligent Electro-Hydraulic Servo Drive Positioning
}

\author{
Edvard Detiček* - Uroš Župerl \\ University of Maribor, Faculty of Mechanical Engineering, Slovenia
}

\begin{abstract}
The goal of the research is to develop a closed loop control system for position control of electrohydraulic servo drive useful for practical application. The research was performed based on the theory of adaptive feedback systems. The proposed new control structure consists of three parts. The first part is actually a feed-forward digital filter in the form of an inverse model of the system, which enables a perfect reference tracking behaviour. The second part is a position feedback fuzzy logic controller designed as a self-learning system, which possesses an ability of automatic tuning and is able to cope with parameter and load changes of the control loop. The third part is the so called switching integrator involved to improve steady state errors and ensure precise final positioning. The effectiveness is proven by laboratory experimental investigations.
\end{abstract}

(C)2011 Journal of Mechanical Engineering. All rights reserved.

Keywords: servo-hydraulic, linear actuators, position control, nonlinear and fuzzy control

\section{INTRODUCTION}

High-speed/high accuracy positioning is a key element in modern mechatronics systems such as computer controlled machine tools, metal-forming machines, manipulators, injection moulding plastic machines, assembly and transport devices, material testing devices, etc. To obtain this rapid movement, hydraulic drives controlled by resistance in impressed pressure network are used very often. In addition to the excellent dynamics, the main advantage of such systems is the parallel use of multiple drives fed by one pressure net combined with energy recycling into the hydraulic accumulators. In particular, the hydraulic cylinders should be stressed at this point due to their ability of direct transformation of hydraulic energy into linear movements and forces. Fluid power actuators are characterized by their high power density and excellent dynamic response. The hydraulic actuators in particular are capable of very high output power levels combined with very compact drive unit dimensions, with very good positioning speed being achieved. They are ideally suited to many high dynamic drive applications in modern machines and mechatronics systems.

The servo-hydraulic cylinder system is also a mechatronic system [1], which can be divided into the electronic part and the mechanical part. It is quite normal nowadays for cylinders to be equipped with electronically controlled proportional and servo valves, as well as with position transducers and force sensors, creating together closed control loops. To fulfil another demand of the above mentioned machines, namely the accurate and precise position and force control, appropriate control strategies are needed. From the control engineering point of view, a good reference tracking for the step, ramp, and sinusoidal reference signals, as well as rejection on external disturbances, have to be achieved.

However, the disadvantages of hydraulic systems such as the low damping ratio, nonlinearities of valve cylinder combination, friction forces, and fluid compressibility need to be overcome. This is successfully obtainable only by implementation of modern control theory and sophisticated digital control algorithms [2]. Backe [2] summarized the development of hydraulic applications from 1975 to 1995 and forecast the need for advanced control techniques.

In other words, to meet the requirements of modern mechatronics systems the electrohydraulic drive systems have to possess increasingly more intelligence. The objective of many researchers is therefore to develop an algorithm which would be able to control the drive without any a-priori knowledge of geometrical, operating or any other parameters of the system. A contribution to achieve the above objective is presented in this paper. A very promising tool to achieve such desired goals is the fuzzy logic control. Fuzzy control technology is based on 
the linguistic variables - the human thought process; the input signal is fuzzified at first, then goes through the fuzzy reasoning process under operational experience and expert knowledge, and finally the control signal is defuzzified and sent out [3] and [4]. In the literature [5] to [7], servo-hydraulic system control has been executed by fuzzy controller successfully; however, which control parameters to use to satisfy the performance requirement is determined through trial and error method. Berger [8] presented the idea of designing fuzzy controllers according to the classification of the controlled systems. Thus, fuzzy controllers can be classified as Fuzzy-PID, Fuzzy-PI and Fuzzy-PD controllers. Yamazaki [9] and Mudi [10] tested the self-tuning of PI controllers using fuzzy logic and demonstrated that fuzzy control was able to reduce the initial cost of building an automatic tuning system. An optimal-tuning PID control has also been devoted to a proportional control system including a 4/3-way proportional valve and a differential cylinder with computer data acquisition system [11] and [12]. Branco [13] has demonstrated the feasibility of using fuzzy control to reduce the influence of unmodeled nonlinearities and parameter uncertainties in hydraulic systems. Jelali [14] summarized recent developments in nonlinear identification, nonlinear control and the application of both to hydraulic servosystems. Kim [15] proposed an experimental optimization algorithm to determine the effective control parameters for an electro-hydraulic position control system. In his algorithm the variations of the system dynamics can be properly compensated. Xiang performed a design study of adaptive Fuzzy PD controller for pneumatic servo-system [16].

This paper presents a new hybrid-fuzzy control strategy for position control of the electrohydraulic linear drive. An adaptability is obtained by fuzzy logic controller designed as a selflearning system, while the reference tracking and position accuracy are improved by conventional control measures such as an inverse feed-forward controller and switching integrator. The algorithm is experimentally investigated and implemented on the hydraulic device for testing mechanical constructions-load simulator.

\section{ADAPTIVE CONTROL STRUCTURE}

There are clear design goals for electrohydraulic drive control system to achieve fast response to step command signals with minimal overshoot (dead-beat response) and approximate zero steady state errors for step, ramp and sinusoidal reference inputs. Those goals have to be obtainable in a rough industrial environment, where significant system behaviour changes may occur due to nonlinear characteristics of system elements, temperature changes, noise

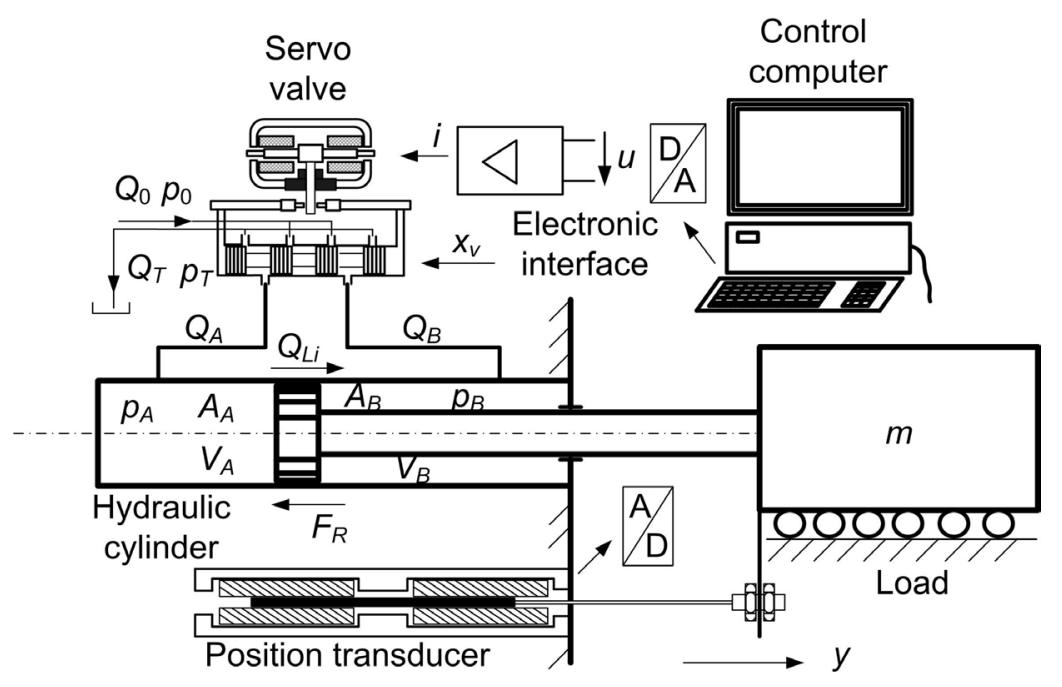

Fig. 1. Shematic presentation of electro-hydraulic servo drive 
and other environmental influences. Therefore, the controller has to be able to adapt itself to the changing properties of controlled process and its signals.

\subsection{Description of Electro-Hydraulic Servo Drive Dynamics}

The schematic of electro-hydraulic servo system is depicted in Fig. 1. It is composed of a single rod cylinder, a 4/3 way servo valve, a sliding load mass, a position transducer, an electronic interface and a control computer. Since the high response servo valve is used it is assumed that the valve spool displacement $x_{V}$ is directly proportional to the electric current $i$ from the electronic amplifier driven by control input voltage $u$.

The relation is described by the following equation where $K_{V}$ is valve spool gain:

$$
x_{V}(t)=K_{V} u(t) \text {. }
$$

The servo valve second stage output flows $Q_{A}, Q_{B}$, by symetric zero overlapping spool and pump flow $Q_{0}$ (rated), supply pressure $p_{0}$, tank pressure $p_{T} \approx 0$, according to Bernoullie's equation are [14]:

$$
\begin{aligned}
& \text { for } x_{V} \geq 0: \\
& Q_{A}=\frac{Q_{0}}{x_{V \max }} x_{V} \operatorname{sign}\left(p_{0}-p_{A}\right) \sqrt{\left|\frac{p_{0}-p_{A}}{p_{0}}\right|}, \\
& Q_{B}=-\frac{Q_{0}}{x_{V \max }} x_{V} \operatorname{sign}\left(p_{B}\right) \sqrt{\left|\frac{p_{B}}{p_{0}}\right|}, \\
& \text { for } x_{V}<0: \\
& Q_{A}=\frac{Q_{0}}{x_{V \max }} x_{V} \operatorname{sign}\left(p_{A}\right) \sqrt{\mid \frac{p_{A}}{p_{0} \mid}}, \\
& Q_{B}=-\frac{Q_{0}}{x_{V \max }} x_{V} \operatorname{sign}\left(p_{0}-p_{B}\right) \sqrt{\left|\frac{p_{0}-p_{B}}{p_{0}}\right|} .
\end{aligned}
$$

Following the continuity condition, with regard to oil compressibility $E(p)$, the pressure changes in both cylinder chambers, can be expressed as:

$$
\begin{aligned}
\frac{d p_{A}(t)}{d t}= & \frac{E\left(p_{A}\right)}{V_{0 A}+A_{A} y(t)}\left[Q_{A}\left(p_{A}, x_{V}, t\right)-\right. \\
& \left.-A_{A} \frac{d y(t)}{d t}-K_{L i}\left(p_{A}(t)-p_{B}(t)\right)\right],
\end{aligned}
$$

$$
\begin{aligned}
\frac{d p_{B}(t)}{d t} & =\frac{E\left(p_{B}\right)}{V_{0 B}-A_{B} y(t)}\left[A_{B} \frac{d y(t)}{d t}-Q_{B}\left(p_{B}, x_{V}, t\right)\right. \\
& \left.+K_{L i}\left(p_{A}(t)-p_{B}(t)\right)\right],
\end{aligned}
$$

where $A_{A}, A_{B}$ are piston areas, $p_{A}$ - left chamber pressure, $p_{B}$ - right chamber pressure, $V_{0 A}, V_{0 B}$ dead volumes, $K_{L i}$ - leakage coefficient, and $y$ is the piston rod position e.g. load position.

The dynamics of the moving parts of the system is expressed by the force equilibrium:

$$
m \frac{d^{2} y}{d t^{2}}=p_{A}(t) A_{A}-p_{B}(t) A_{B}-F_{R}(t)-F_{L}(t),
$$

where $m$ is the moving mass, $F_{R}(t)$ is friction force and $F_{L}(t)$ is external load force.

Finally, by rearrangement of the above equations and introduction of some new terms, such as velocity gain $V_{S}\left(p_{A}, p_{B}, y\right)$, eigen frequency $\omega\left(p_{A}, p_{B}, y\right)$ and damping $D\left(p_{A}, p_{B}, y\right)$, leads to a common mathematical expression:

$$
\begin{aligned}
& \frac{d^{3} y(t)}{d t^{3}}+2 D\left(p_{A}, p_{B}, y\right) \omega\left(p_{A}, p_{B}, y\right) \frac{d^{2} y(t)}{d t^{2}}+ \\
& +\omega^{2}\left(p_{A}, p_{B}, y\right) \frac{d y(t)}{d t}=\omega^{2}\left(p_{A}, p_{B}, y\right) . \\
& \cdot V_{s}\left(p_{A}, p_{B}, y\right) K_{V} u(t)-\frac{d F_{L}}{d t m} .
\end{aligned}
$$

After linearization of Eq. (6) in particular operating point, using Laplace transformation, the transfer function of the entire system can be derived. It generally has the form of a third order system (second order system and integrator), which numerical parameter varies among different operating points. A major simplification is done by neglecting the second order term (neglected oil compressibility and inertial forces) and representing the system by transfer function of an integrator:

$$
G(s)=\frac{y(s)}{u(s)}=\frac{V_{S} K_{V}}{s}=\frac{K_{I}}{s} .
$$

It should be pointed out that integrator gain $K_{I}$ significantly depends on piston movement direction.

\subsection{Adaptive Control Structure}

A successfully applied control strategy based on conventional control theory is depicted 
in Fig. 2. It consists of a digital self-tuning, parameter adaptive controller to cope with parameter changes, a cancellation feed-forward controller to minimize steady-state errors of ramp and sinusoidal reference inputs and a switching integrator to improve precise final positioning of hydraulic drive.

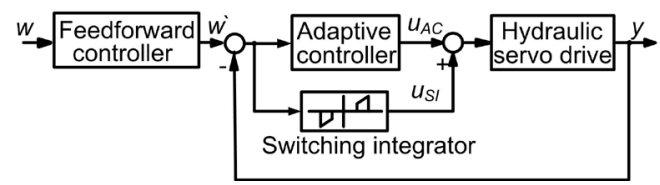

Fig. 2. Adaptive control structure for position control of electro-hydraulic drive

It is important to obtain the digital parameter adaptive controller, to be realizable on a control computer, inside a sampling interval, which needs to be small enough to represent electro-hydraulic servo drive dynamics well (in our case $T=2 \mathrm{~ms}$ ). Therefore, the self-tuning approach is used and a simplified mathematical model Eq. (7) is taken to be the identification model of the system. However, it shows that all changes are reflected on load pressure changes and therefore on $K_{I}$ as well. In that way it is the function, not only of systems state variables, servo valve rated flow, piston area and supply pressure but also of inertial, friction and external forces. Putting Eq. (7) in to discrete form it gives:

$$
G_{m}(z)=\left(1-z^{-1}\right) z\left(\frac{K_{I}}{s^{2}}\right)=\frac{T K_{I}}{1-z^{-1}}=\frac{b_{1}}{1-z^{-1}} .
$$

Therefore, a realizable, digital parameter adaptive (self tunning) controller, with only one unknown parameter $\hat{b}_{1}$ is obtained using recursive least square estimation (Iserm). The equation for unknown parameter vector estimation has the general form:

$$
\widehat{\widehat{\Theta}}(k+1)=\underline{\widehat{\Theta}}(k)+\underline{\gamma}(k) e(k+1),
$$

with correction vector $\gamma(k)$ :

$$
\gamma(k)=\frac{\underline{P}(k) \underline{\Psi}(k+1)}{\underline{\Psi^{T}}(k+1) \underline{P}(k) \underline{\Psi}(k+1)+\lambda(k+1)},
$$

where $\Psi$ is the signal vector and $P$ is the covariance matrix, with $\lambda$ - forgetting factor:

$$
\underline{P}(k+1)=1 / \lambda(k+1)\left[\underline{I}-\underline{\gamma}(k) \underline{\Psi^{T}}(k+1)\right] \underline{P}(k) .
$$

The final form of an adaptive $P$-controller with initial model value $b_{m}$ (Koek) is:

$$
u_{A C}(k)=\frac{b_{m}(k)}{\hat{b}_{1}(k)} \quad, \widehat{b}_{1}(k)=\left\{\begin{array}{l}
\widehat{b}_{1}(k), \quad \hat{b}_{1}(k) \geq \widehat{b}_{1 \min } \\
\hat{b}_{1}(k-1), \quad \text { else }
\end{array}\right.
$$

The computer control algorithm also contains a decision logic to separate computing of $\left(b_{m, \text { left }}, \hat{b}_{1, \text { left }}, b_{m, \text { right }}, \hat{b}_{1, \text { right }}\right)$, depending on the movement direction. The effectiveness of the above adaptive P-controller is experimentally proven by step change of supply pressure (see Fig. 9). Supply pressure change has been avoided from any further test, while this is not a part of normal operating conditions.

The feed-forward controller (UNbeha) is realized on the basis of the inverse model $G_{m}{ }^{-1}(z)$. It has non-causal form but is realizable in control computer for a partial compensation of steadystate errors by ramp and sinusoidal reference signals:

$$
w^{\prime}(k)=\frac{1}{b_{m}}[w(k+1)+w(k)] .
$$

It also depends on movement direction $\left(b_{m, l e f t}, b_{m, r i g h t}\right)$. Switching integrator in form:

$$
u_{S I}(k)=\left\{\begin{array}{cc}
u_{S I}(k-1)+\Delta u_{S I}(k), & \text { if } e_{\min } \leq e(k) \leq e_{\max } \\
0, & \text { else }
\end{array}\right.
$$

is switched on to improve final position accuracy. It is switched off just before the desired position arrives to prevent limit cycle oscillations.

Among the several advantages of the above control structure the main disadvantage is the inability to obtain automatic first start because the initial value of the estimated parameter must be known in advance.

To overcome this problem our further investigations lead to the replacement of the conventional adaptive controller by appropriate fuzzy logic self learning (self organizing) algorithm.

\section{FUZZY POSITION CONTROL OF ELECTRO-HYDRAULIC LINEAR DRIVE}

\subsection{Fuzzy Control}

Fuzzy logic, developed by [3] in 1960s, is much closer to human reasoning and natural 
language. In contrast to the traditional set theory, where elements are only, either complete members of a set, or complete non-members of a set. It provides an effective means of capturing the approximate and inexact nature of the real world. Fuzzy sets have no precise criterion for membership.

To distinguish between members that are more probable than those which are less probable in belonging to the set, the grade of membership denoted by $\mu$ which lies in the range of 0 to 1 is used. The fuzzy sets can have different shapes such as triangular, trapezoidal, bell-shape, etc. The elements of the fuzzy set are taken from the universe of all elements.

A principal characteristic of fuzzy control is that it works according to linguistic rules such as "if the room temperature is low then increase heating" rather than obeying mathematical models and functional relationship. The fuzzy controller consists of four main elements: a fuzzifier, an inference engine, a rule base and a defuzzifier.

The first step of the fuzzy controller design is to select the number of controller inputs and corresponding number of input membership functions required for the fuzzification process. The fuzzification is the process of translating crisp input values into fuzzy linguistic values through the use of membership functions. In other words, determining how much each real value belongs to each fuzzy set using the corresponding membership value.

The fuzzy rule base is simply a data base of the desired control rules for the system. It is most equivalent to the controller of a traditional control system and formalizes the designer's "expert knowledge" of what control output should result from a given combination of system states, expressed in a linguistic manner. It often takes the form of a truth table consisting of rules constructed in the following form: IF "condition 1" AND/ OR “condition 2" THEN “consequence 1". In fuzzy logic terminology, the statement following the IF condition is known as the "premise", "antecedent" or "condition". The corresponding statement following THEN is known as the "conclusion" or "consequent". The inference engine is the heart of a fuzzy logic controller. It acts as the bridge between the fuzzification input stage and defuzzification output stage of the controller, translating the designer's desired control rules from linguistic representation to a numeric computation. The inference engine can be divided into three elements. The first step is known as "aggregation". During this step the premise (IF statement) of each rule in the rule base is calculated using the fuzzified controller inputs. With fuzzification, each condition in the premise is assigned a degree of membership in the corresponding input fuzzy set. It should be noted that the traditional formulation of logical statements such as AND/OR has been modified to accommodate the use of membership functions. In particular, the result of an AND operation is often defined as either the minimum or a product of two fuzzy values compared. Similarly, an OR operation is often defined as either the maximum or probabilistic sum. Any premise with a value greater than zero means that its corresponding rule is active. The second step of the inference process is known as "composition" or "implication", where the consequent (THEN statement) of each rule is created using the premises calculated in the first step. The output of the composition step is not a single value for each rule in the rule base, but rather one modified output fuzzy set for each rule. These modified output sets are known as "implied" fuzzy sets. The modification is controlled by the premise calculated in the aggregation step. There are two fundamental methods of creating the fuzzy sets that are the results of composition min and prod. The min operation truncates the output fuzzy set at the value of the premise while the prod operation scales the output fuzzy set according to the premise. The third and final step of the inference process is known as "accumulation" or "results aggregation". In this step the implied fuzzy sets that are the output of the composition process are combined into an accumulated fuzzy set, which is the input to the defuzzification. Defuzzification is the process of converting the fuzzy output set which is the result of inference of the process into a discrete number. Two fundamental methods are known as the Mean of Maxima and the Center of gravity. The latter, also known as the Center of Area method involves two steps:

- multiply the membership degree $\mu\left(x_{i}\right)$ of each element $i$ by the singleton value $x_{i}$ of the membership function. 
- $\quad$ sum the values obtained in the first step and divide total by the sum of the output membership degrees.

\subsection{Fuzzy PD Controller}

For stability reasons the general form of PD-controller was used at the beginning:

$$
u=f(e, \dot{e}) \text {. }
$$

By fuzzification of two input variables $(e, \dot{e})$ and one output variable $(u)$ of the controller, a two- dimensional rule base has been constructed. Linguistic variables $e, \dot{e}$, and $u$ are shown in Fig. 3. The universe of discourse of each variable is selected in range 1: $\alpha$, where $\alpha=A_{A} / A_{B}$ is piston area ratio.

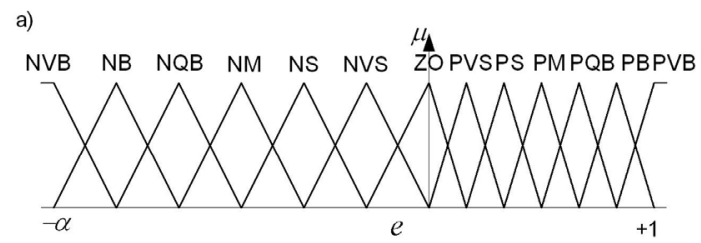

b)

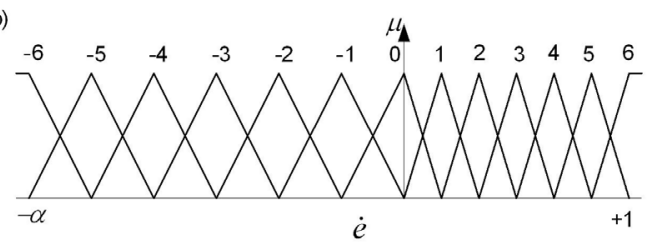

c)

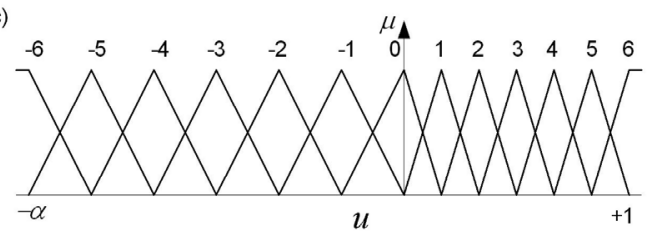

Fig. 3. Linguistic variables represented as fuzzy variables; a) e, b) $\dot{e}, c) u$

The rule base or decision table, shown in Fig. 4, contains linguistic rules of the form: IF (A and B ) THEN C. In the present context the antecedents $\mathrm{A}$ and $\mathrm{B}$ refer to linguistic statements about the error between set point and actual position of the moving mass and the rate of change of this error. The logic conclusion of each rule is about the servo valve opening. Each non zero entry in the decision table corresponds to one rule. Two axes or universes of discourse of the decision table are for error $e$ and its change $\dot{e}$ respectively. Each axis has 13 entries from -6 through 0 to +6 . The points 1 to 6 can be taken to mean: very small, small, medium, quite large and very large. The fuzzy sets have, for computational simplicity, a triangular shape with $\mu$ - values of 0 , $0.1,0.2, \ldots, 0.9,1$. The consequent of each rule in the matrix also has one of 13 values and represents the contribution of each rule to the output action set. The collective effect of all rules can be given as: (rule 1) ELSE (rule 2) ELSE (rule 3) ELSE... "ELSE" can be regarded as an inclusive OR function or MAX function. Finally, the centre of gravity of the output action set was used, as the method of defuzzification, to obtain unique real output.

From Fig. 4 it can be seen that the rule base is diagonally symmetrical and the rules along the diagonal change equally.

\begin{tabular}{|c|c|c|c|c|c|c|c|c|c|c|c|c|c|}
\hline & \multicolumn{13}{|c|}{$e(k)$} \\
\hline & -6 & -5 & -4 & -3 & -2 & -1 & 0 & 1 & 2 & 3 & 4 & 5 & 6 \\
\hline-6 & -6 & -6 & -6 & -6 & -6 & -5 & -4 & -3 & -2 & -1 & 0 & 0 & 0 \\
\hline-5 & -6 & -6 & -6 & -5 & -5 & -4 & -3 & -2 & -1 & 0 & 0 & 0 & 0 \\
\hline-4 & -6 & -6 & -5 & -5 & -4 & -3 & -2 & -1 & 0 & 0 & 0 & 0 & 1 \\
\hline-3 & -6 & -5 & -5 & -4 & -3 & -2 & -1 & 0 & 0 & 0 & 0 & 1 & 2 \\
\hline-2 & -6 & -5 & -4 & -3 & -2 & -1 & -1 & 0 & 0 & 0 & 1 & 2 & 3 \\
\hline-1 & -6 & -4 & -3 & -2 & -1 & -1 & 0 & 0 & 0 & 1 & 2 & 3 & 4 \\
\hline$\dot{e}(k) \quad 0$ & -6 & -4 & -3 & -2 & -1 & -1 & 0 & 1 & 1 & 2 & 3 & 4 & 6 \\
\hline 1 & \begin{tabular}{|l|}
-4 \\
\end{tabular} & -3 & -2 & -1 & 0 & 0 & 0 & 1 & 1 & 2 & 3 & 4 & 6 \\
\hline 2 & -3 & -2 & -1 & 0 & 0 & 0 & 1 & 1 & 2 & 3 & 4 & 5 & 6 \\
\hline 3 & \begin{tabular}{|l|}
-2 \\
\end{tabular} & -1 & 0 & 0 & 0 & 0 & 1 & 2 & 3 & 4 & 5 & 5 & 6 \\
\hline 4 & -1 & 0 & 0 & 0 & 0 & 1 & 2 & 3 & 4 & 5 & 5 & 6 & 6 \\
\hline 5 & 0 & 0 & 0 & 0 & 1 & 2 & 3 & 4 & 5 & 5 & 6 & 6 & 6 \\
\hline 6 & 0 & 0 & 0 & 1 & 2 & 3 & 4 & 5 & 6 & 6 & 6 & 6 & 6 \\
\hline
\end{tabular}

Fig. 4. Rule base or decision table

The consequence is a linear dynamic behaviour of the controller. In other words, the fuzzy controller emulates an exactly conventional PD-controller. This was practically proved by experiments. Due to fuzzyffication of measurement signals and robustness of fuzzy rules, the fuzzy controller is less sensitive to noise and small parameter variations. It is therefore, to a certain extent, more robust than the conventional one. 
a)

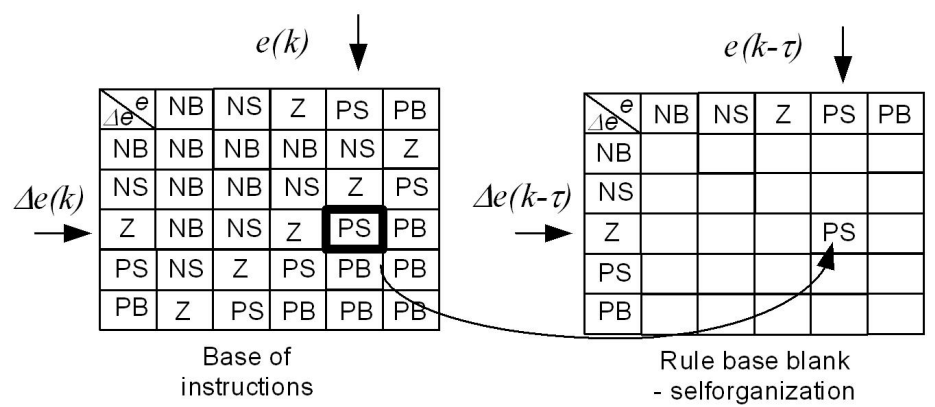

PS and blank is $P S$

b)

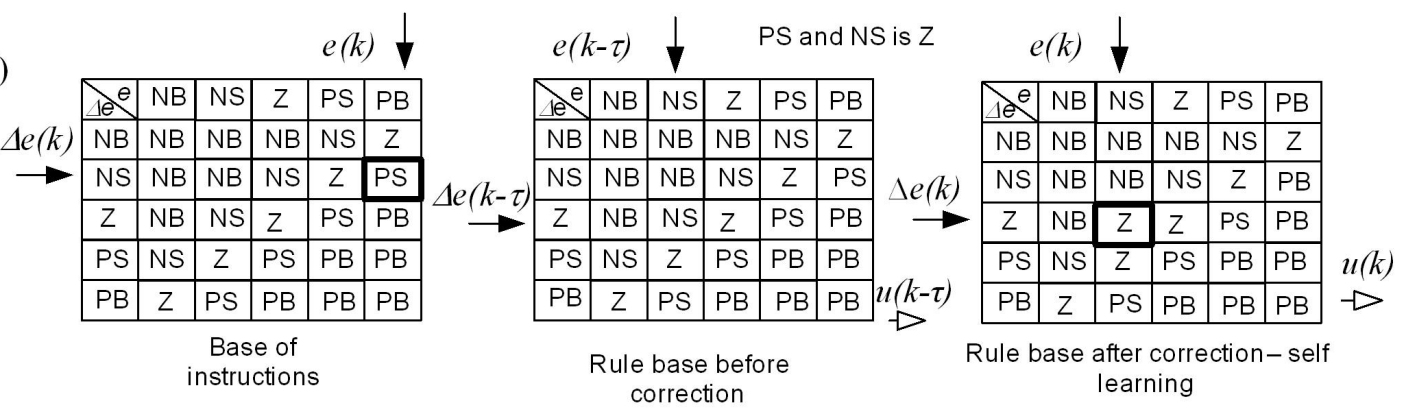

Fig. 5. a) Self-organizing mechanism of fuzzy logic controller; b) Self-learning mechanism of fuzzy logic controller

On the other hand, it is possible to obtain nonlinear characteristics of the fuzzy PDcontroller by modification of the symmetrical rule base.

The following paragraph describes an attempt of automatic rule base creation.

\subsection{Fuzzy Self-Learning Controller}

The theory of fuzzy control seems to be a suitable mathematical tool for both modelling and control of complex systems [3]. However, the primitive form of fuzzy control sometimes fails in dealing with these complex systems mainly because it lacks enough adaptability. Several researchers have devoted themselves to developing fuzzy controller with more "intelligence" [1], [17] and [18]. Unfortunately, most of them are difficult for real time implementation.

Therefore, we decided to implement the so called reinforcement learning, proposed in [9] and [19].

Self-learning fuzzy logic controller tries to emulate human decision making behaviour and learning, namely, an ability to make rules and to modify them based on experience. It has a hierarchical structure, which consists of two rule bases.

The first one is constructed as a base of instructions and a base of performances simultaneously. It creates and modifies the main rule base according to the desired overall performance of the system. At the first start of the controller, the main rule base contains zero values and it begins to fill according to base of instructions.

The quality of control action is checked by a comparison of present values of control error $e$ and $\dot{e}$ with expected values from the base of performances. The linguistic terms in the base of performances show how the rules must be changed. The question is which rule must be changed, if we know that the present $e$ and $\dot{e}$ are caused by a past control action. The solution is determining the time constant $\tau$, which express that the control result at $n^{\text {th }}$ discrete moment of time is a consequence of control action activated at the $(n-\tau)^{\text {th }}$ discrete moment in the past. Determination of $\tau$ is done on the base of heuristic knowledge of dynamic behaviour of the control process. As the investigations show, the selection of the $\tau$ is not critical and can be made inside a certain range of values. The self learning fuzzy controller 
therefore, uses control computer memory buffer to save past information and contains the mechanism for changing rules (Fig. 5a) or creating new rules (Fig. 5b), if they do not yet exist. It can be said that the controller also possesses the ability to self organize.

Let us suppose the above mentioned learning process is convergent, then the general rule base become constant after certain number of experiments. As long as the changes in system parameters are not significant, the rule base stays constant; otherwise a new adaptation is needed.

The self-learning and self-organizing mechanism is explained comprehensively in Fig. 5 , with only three linguistic terms for the sake of simplicity.

a)

$\begin{array}{llllllllllllll}-6 & -5 & -4 & -3 & -2 & -1 & 0 & 1 & 2 & 3 & 4 & 5 & 6\end{array}$

\begin{tabular}{l|l|l|l|l|l|l|l|l|l|l|l|l|l|}
\hline 0 & 0 & 0 & 0 & 0 & 0 & 0 & 0 & 0 & 0 & 0 & 0 & 0 & 0 \\
\hline
\end{tabular}

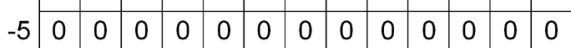

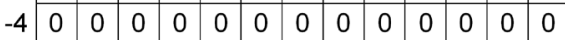

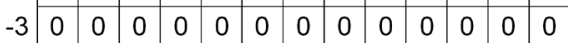

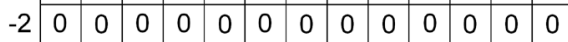

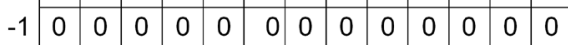

$\begin{array}{llllllllllllllll}0 & 0 & 0 & 0 & 0 & 0 & 0 & 0 & 0 & 0 & 0 & 0 & 0 & 0\end{array}$

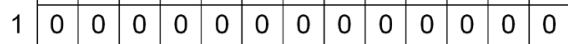

2 \begin{tabular}{llllllllllllllllll|}
\hline & 0 & 0 & 0 & 0 & 0 & 0 & 0 & 0 & 0 & 0 & 0 & 0 & 0
\end{tabular}

\begin{tabular}{llllllllllllllllll|}
3 & 0 & 0 & 0 & 0 & 0 & 0 & 0 & 0 & 0 & 0 & 0 & 0 & 0
\end{tabular}

4 \begin{tabular}{l|l|l|l|l|l|l|l|l|l|l|l|l|}
\hline 0 & 0 & 0 & 0 & 0 & 0 & 0 & 0 & 0 & 0 & 0 & 0 & 0
\end{tabular}

\begin{tabular}{l|l|l|l|l|l|l|l|l|l|l|l|l|l|}
5 & 0 & 0 & 0 & 0 & 0 & 0 & 0 & 0 & 0 & 0 & 0 & 0 & 0 \\
\cline { 2 - 11 } 6 & 0 & 0 & 0 & 0 & 0 & 0 & 0 & 0 & 0 & 0 & 0 & 0 & 0 \\
\hline
\end{tabular}

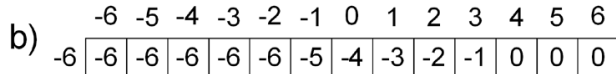

\begin{tabular}{ll|l|l|l|l|l|l|l|l|l|l|l|l|}
-5 & -6 & -6 & -6 & -5 & -5 & -4 & -3 & -2 & -1 & 0 & 0 & 0 & 0 \\
\hline
\end{tabular}

\begin{tabular}{lllllllllllllllllll}
-4 & -6 & -6 & -5 & -5 & -4 & -3 & -2 & -1 & 0 & 0 & 0 & 0 & 1 \\
\hline
\end{tabular}

\begin{tabular}{llllllllllllllllllll}
-3 & -6 & -5 & -5 & -4 & -3 & -2 & -1 & 0 & 0 & 0 & 0 & 1 & 2 \\
\hline
\end{tabular}

\begin{tabular}{lllllllllllllllllll|}
-2 & -6 & -5 & -4 & -3 & -2 & -1 & -1 & 0 & 0 & 0 & 1 & 2 & 3 \\
\hline
\end{tabular}

\begin{tabular}{llllllllllllllllllll}
-1 & -6 & -4 & -3 & -2 & -1 & -1 & 0 & 0 & 0 & 1 & 2 & 3 & 4 \\
\hline
\end{tabular}

\begin{tabular}{lllllllllllllllllll|}
\hline 0 & -6 & -4 & -3 & -2 & -1 & -1 & 0 & 1 & 1 & 2 & 3 & 4 & 6 \\
\hline
\end{tabular}

\begin{tabular}{ll|l|l|l|l|l|l|l|l|l|l|l|l|}
1 & -4 & -3 & -2 & -1 & 0 & 0 & 0 & 1 & 1 & 2 & 3 & 4 & 6
\end{tabular}

\begin{tabular}{llllllllllllllllll|}
2 & -3 & -2 & -1 & 0 & 0 & 0 & 1 & 1 & 2 & 3 & 4 & 5 & 6 \\
\hline
\end{tabular}

\begin{tabular}{ll|l|l|l|l|l|l|l|l|l|l|l|l|}
3 & -2 & -1 & 0 & 0 & 0 & 0 & 1 & 2 & 3 & 4 & 5 & 5 & 6
\end{tabular}

\begin{tabular}{lllllllllllllllll|l|l|}
4 & -1 & 0 & 0 & 0 & 0 & 1 & 2 & 3 & 4 & 5 & 5 & 6 & 6 \\
\hline
\end{tabular}

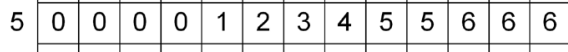

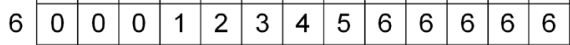

Fig. 6. a) Rule base or decision table, b) base of instructions
Fig. 6 shows the original forms of rule base and base of instructions at the beginning of the control process. The rule base and the base of instructions has integer entries ranging from -6 to +6 . These entries are positive or negative reinforcements, which are given to the corresponding $(e, \dot{e})$ pair in the rule base.

Reinforcements also implicate rules creation, in case they do not yet exist. Fig. 6b shows the matrix developed by [9], although the other versions could be used. Learning proceeds as follows. Let us assume a delay of $\tau$ samples (in our case $\tau=4$ ) on controlled system, then the present $e$ and $\dot{e}$ values are mainly the consequences of controller output from $\tau$ samples ago. Each past state consisting of a triplet $\left(e_{\tau}, \dot{e}_{\tau}, u_{\tau}\right)$ is stored in a control computer "history" buffer. As the present values of $e$ and $\dot{e}$ point to the value $p$ in the performance matrix, then the rule entry for $e_{\tau}, \dot{e}_{\tau}$ in the rule base become $u_{\tau},+p$, whatever the rule entry may have been up to the present time.

The self-organizing controller comprises both the blank rule base fuzzy controller and the above learning process.

\section{EXPERIMENTAL RESULTS}

The laboratory experiments were performed on laboratory testing equipment shown in Fig. 7. First the self tuning parametric adaptive controller, according to Eq. (12), was tested. It was compared with an ordinary P-controller (Fig. 8a), by ramp reference signals.

Adaptive ability was proven by a sudden supply pressure drop of $20 \%$, at the time of 0.3 s (Fig. 8b). The next experiments were step responses of fuzzy logic controller. First the selforganizing fuzzy logic controller was started with zero rule base (Fig. 9a), while the learning process was currently involved in the following tests (Fig. 9b).

The rule base becomes mostly constant after the second and third step. Finally such an "experienced" fuzzy controller, together with feed-forward controller Eq. (13) and switching integrator Eq. (14) were joined in, so called, "hybrid" control structure to obtain a ramp response of the hydraulic drive (Fig. 10). 

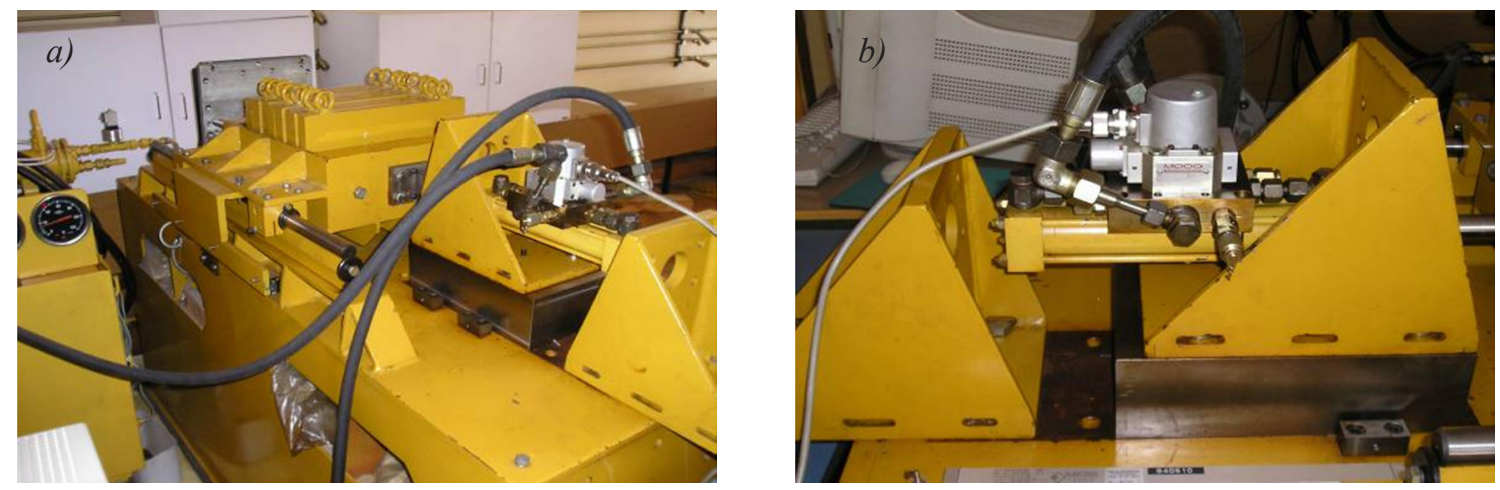

Fig. 7. a) Structure of the testing system (cylinder $40 / 28 \mathrm{~mm}$, slider mass 70 to $300 \mathrm{~kg}$, lin. pot. $300 \mathrm{~mm}$ $\pm 1 \%$ ), b) Servo valve (MOOGD-769-233, $Q=19 \mathrm{l} / \mathrm{min}$ )
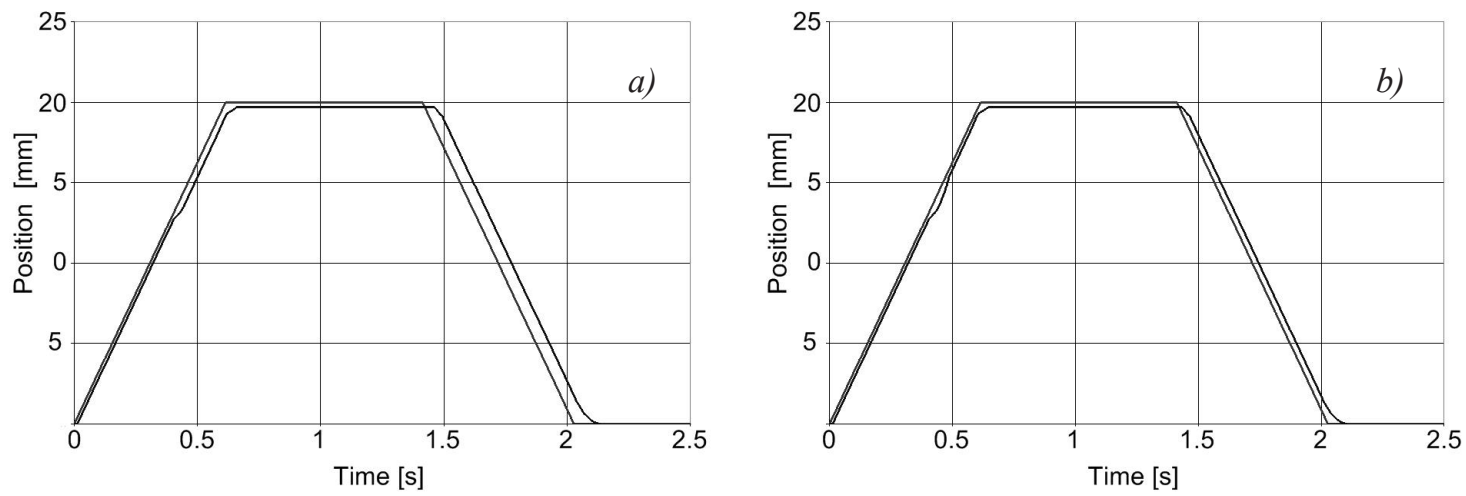

Fig. 8. a) P-controller ramp response by 20\% supply pressure drop, b) Self-tuning controller ramp response by $20 \%$ supply pressure drop

a)

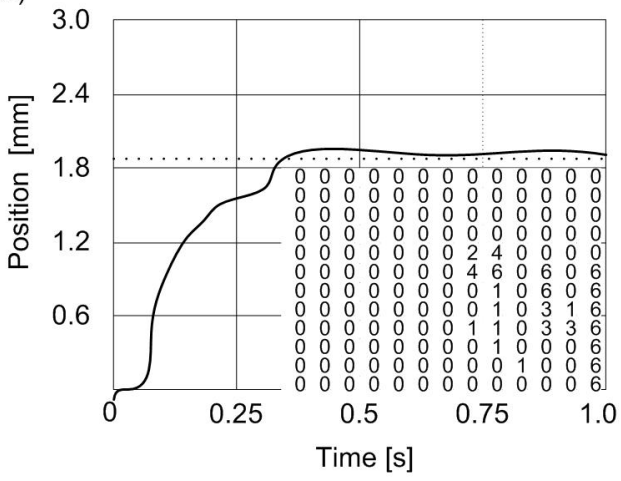

b)

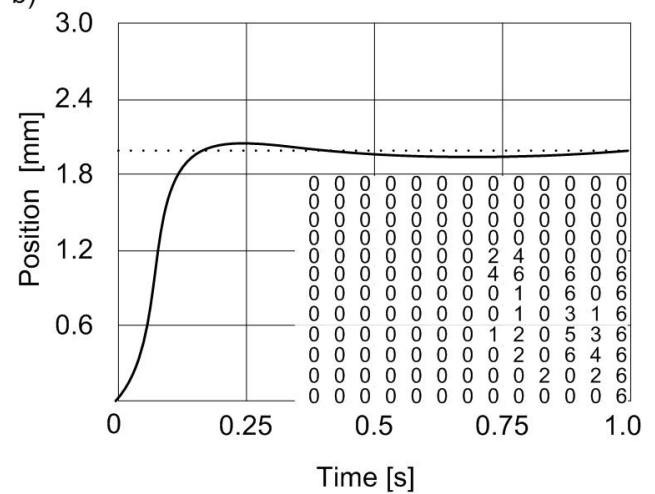

Fig. 9. a) First step of learning process, b) Third step of learning process

The proposed hybrid fuzzy control structure is shown in Fig. 11.

The experiments show that the selforganizing fuzzy controller enables a successful control of electro-hydraulic drive without any a-prior knowledge of the system dynamics in the first starting conditions [20] and [21]. It is also able to cope with non-linearity and parameter changes and therefore, possess a certain level of adaptability as well. However, the reference tracking behaviour [22] and final positioning accuracy are still poor. 


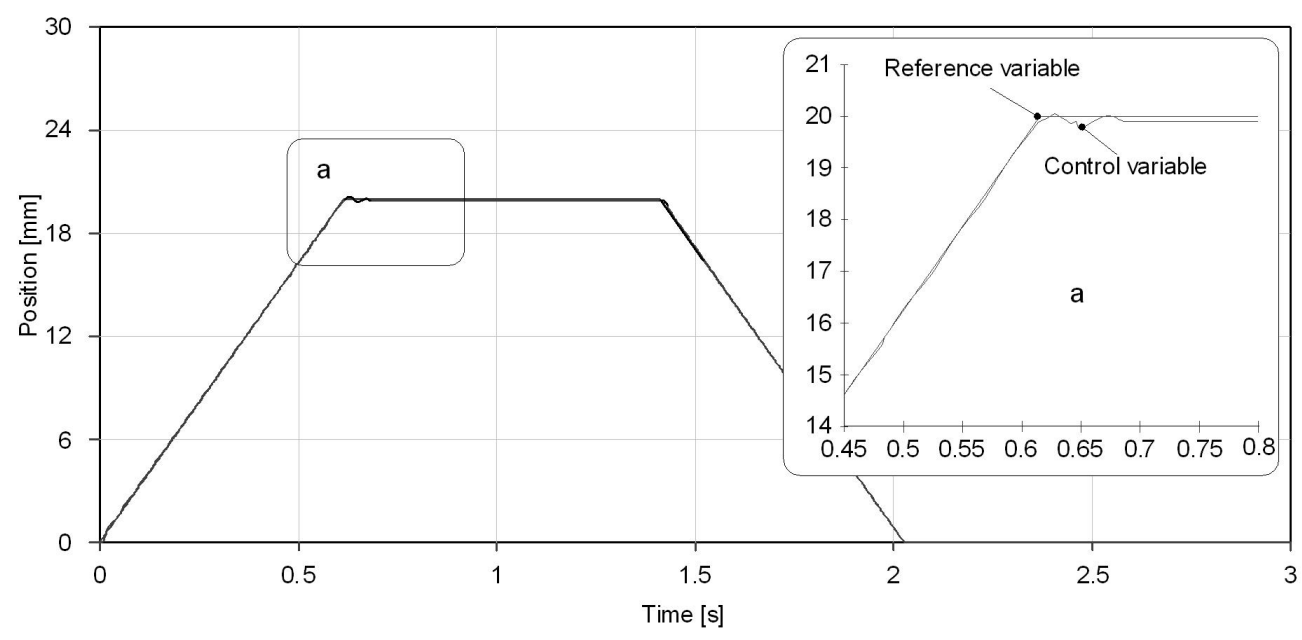

Fig. 10. Ramp response of electro-hydraulic servo drive with hybrid fuzzy position controller

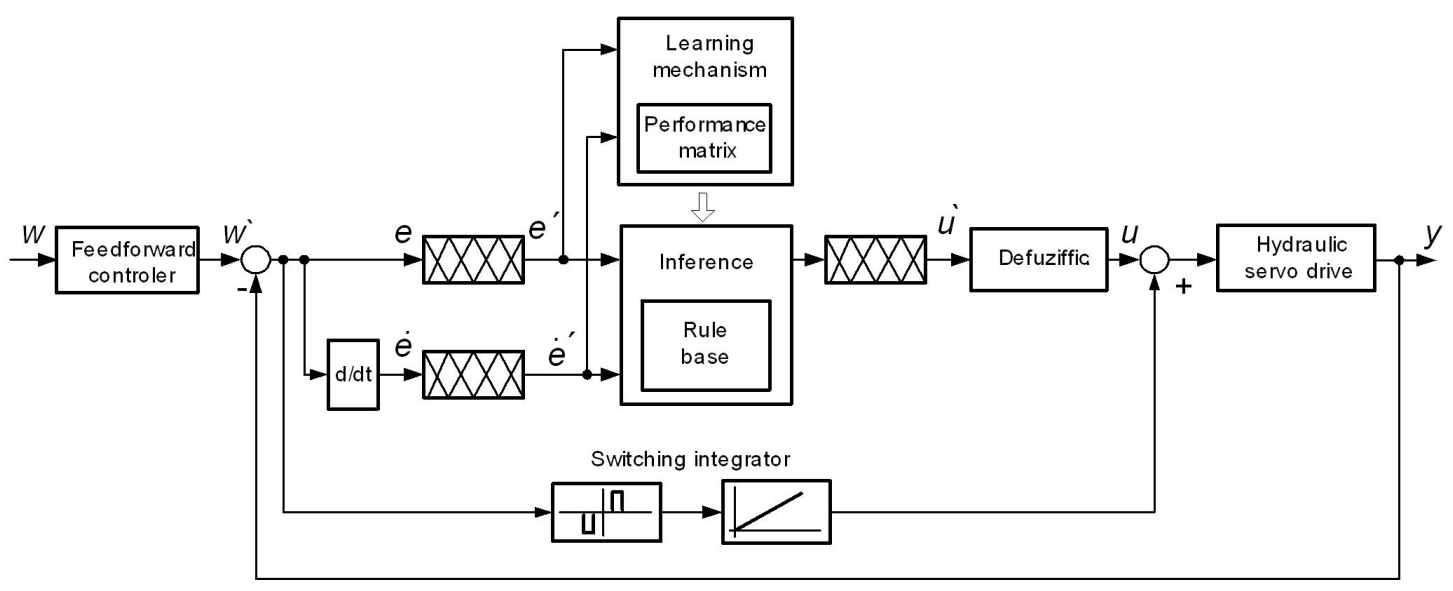

Fig. 11. Hybrid fuzzy control structure of electro-hydraulic servo drive position control

\section{CONCLUSIONS}

Systems having structural uncertainties or a known complicated structure such as hydraulic systems are difficult to control.

The dynamic characteristics of such systems are usually very complex and highly nonlinear. For a practical control system, it is usually desired to have a fast accurate response with a small over-shoot.

In this paper, a possibility to apply a selforganising and self learning fuzzy algorithm for position control of hydraulic servo drive is represented. In addition to self-organisation it also possesses the ability of self learning. It is able of adaptation to parameter changes and to therefore, deal with nonlinear dynamic behaviour associated with the drive motion.

Unfortunately, there is no systematic way to prove the convergence of a learning mechanism and overall stability of the control system. Trial and error method was used with these experiments. Therefore, additional research will be required in the future.

\section{REFERENCES}

[1] Shih, M.C., Tsai, C.P. (1995). Servohydraulic cylinder position control using a neuro-fuzzy 
controller. Mechatronics, vol. 5, no. 5, p. 497512.

[2] Backe, W. (1993). The present and future of fluid power. Proceedings of the Institution of Mechanical Engineers, Part I: Journal of Systems and Control Engineering, vol. 207, p. 193-212.

[3] Zadeh, L.A. (1965). Fuzzy Sets, Informat Control, vol. 4, no. 8, p. 126-174.

[4] Bouslama, F., Ichikawa, A. (1992). Fuzzy control rules and their natural control laws. Fuzzy Sets Syst., vol. 48, p. 65-86.

[5] Shih, M.C., Chen, P.C. (1991). An experimental study on the position control of a hydraulic cylinder usinga fuzzy logic controller. JSME, Series III, vol. 34, no. 4, p. 481-489.

[6] Chen, C.Y., Liu, L.Q., Cheng, C.C., George, T. (2008). Fuzzy controller design for synchronous motion in a dualcylinder electro-hydraulic system. Control Engineering Practice, vol. 16, no. 6, p. 658673.

[7] Kastrevc, M., Pusenjak, R. (2005). Fuzzy pressure control of hydraulic system with gear pump driven by variable speed induction electro motor. Exp. Tech. (Westport Comm.), vol. 29, no. 3, p. 57-62.

[8] Berger, M. (1996). Self-tuning of a PI controller using fuzzy logic for a construction unit testing apparatus. Control Engineering Practice, vol. 4, no. 6, p. 785-790.

[9] Yamazaki, T. (1982). An improved algorithm for self-organising controller and its experimental analisys. John Hopkins, University Press, Baltimore.

[10] Mudi, R.K., Pal, N.K. (2000). A self-tuning fuzzy PI controller. Fuzzy Sets Systems, vol. 115, no. 2, p. 327-338.

[11] Liu, G.P., Daley, S. (2000). Optimal-tuning nonlinear PID control of hydraulic systems. Control Engineering Practice, vol. 8, no. 9, p. 1045-1053.

[12] Kim, S.M., Han, W.Y. (2006). Induction motor servo drive using robust PID-like neuro-fuzzy controller. Control Engineering Practice, vol. 14, no. 5, p. 481-487.

[13] Branco, P.J.C., Dente, J.A. (2000). On using fuzzy logic to integrate learning mechanisms in an electro-hydraulic system - Part I: Actuator's fuzzy modeling. IEEE Transactions on Systems, Man, and Cybernetics - Part C: Applications and Reviews, vol. 30, no. 3, p. 305-316.

[14] Jelali, M., Kroll, A. (2003). Hydraulic servosystem modeling, identification and control. Springer, London, New York.

[15] Kim, M.Y., Lee, C.O. (2006). An experimental study on the optimization of controller gains for an electro-hydraulic servo system using evolution strategies. Control Engineering Practice, vol. 14, no. 2, p. 127-147.

[16] Xiang, G., Zheng-Jin, F. (2005). Design study of an adaptive Fuzzy-PD controller for pneumatic servo system. Control Engineering Practice, vol. 13, no. 1, p. 55-65.

[17] Čuš, F., Župerl, U. (2007). Adaptive selflearning controller design for feedrate maximization of machining process. Advances in Production Engineering \& Management, vol. 2, no. 1, p. 18-27.

[18] Milfelner, M., Župerl, U., Čuš, F. (2004). Optimisation of cutting parameters in high speed milling process by GA. International Journal of Simulation Modelling, vol. 3, no. 4, p. 121-131.

[19]Lee, C.C. (1990). Fuzzy logic in control systems. IEEE Transaction on Systems, Man and Cybernetics, vol. 20, no. 2, p. 24-32.

[20] Herakovič, N. (1995). Piezoaktorbetätigung für ein einstufiges hochdynamisches Servoventil $=[$ Piezoactuator for a singlestage servovalve with high dynamic response]. $O+P$, Ölhydraul. Pneum., vol. 39, no. 8, p. 601-605.

[21]Župerl, U., Čuš, F., Kiker, E., Milfelner, M. (2005). A combined system for off-line optimization and adaptive adjustment of the cutting parameters during a ball-end milling process. Strojniški vestnik - Journal of Mechanical Engineering, vol. 51, no. 9, p. 542-559.

[22] Roy, S.S. (2010). Modelling of tool life, torque and trust force in drilling: a neurofuzzy approach. International Journal of Simulation and Modelling, vol. 9, no. 2, p. 74-85. 